\title{
Estudio de blindaje magnético en materiales conductores en función de la frecuencia
}

\author{
Study of Magnetic Shielding in Conductors Materials as a Function of the \\ Frequency
}

\author{
J. A. Torres Velásquez ${ }^{a, *}$ \\ R. R. Viáfara Avila ${ }^{a}$ \\ C. A. Chacón Cardona ${ }^{\mathrm{a}}$
}

Recepción: 26-may-2015

Aceptación: 13-oct-2015

\section{Resumen}

Se describe el efecto de un campo magnético uniforme en materiales conductores, y cómo estos apantallan el campo que los atraviesa. Con el fin de crear dicho campo,se energiza una bobina que encierra el material apantallador y la bobina sensor de campo magnético, mediante una señal sinusoidal producida por un generador de señales con una tensión de 10 [Vp]. La medida del campo magnético se realiza de manera indirecta ubicando una bobina como sensor en el centro de una bobina de diámetro mayor, realizando la medida de tensión en el sensor, de tal manera que al colocar un material apantallador que encierre la bobina se reduzca el campo magnético en su interior y, por ende, la tensión inducida en la bobina. Los materiales apantalladores que se emplearon fueron: acero, aluminio, hierro y cobre; la eficiencia de estos materiales fue estudiada en un rango de frecuencias de $60-100.000 \mathrm{~Hz}$, obteniendo como resultado que el material más eficiente desde 60- $12.000 \mathrm{~Hz}$ es el hierro, y para 32.000-100.000 es el cobre.

Palabras clave: apantallamiento magnético, campo magnético, eficiencia, tensión, frecuencia.

\begin{abstract}
This study describes the effect of a uniform magnetic field in conductive materials, and how they shield the magnetic field that travel through them. In order to create such a field, two coilis are energized by a sinusoidal signal produced by a signal generator with a difference of potential of 10 [Vp]. The magnetic field measurement is performed indirectly by placing a coil as a sensor in the center of a larger diameter coil, performing the tension difference of potential measurement on the sensor,in such way that by placing a shielding material that encloses the coil and its sensor, the magnetic field inside is reduced, therefore also diminishes the induced tension in the coil. The shielding materials used were steel, aluminum, iron and copper; the efficiency of these materials was studied in a frequency range from 60 to $100.000 \mathrm{~Hz}$, resulting that iron is more efficient than the others from 60 to $12.000 \mathrm{~Hz}$, and for $32.000-100.000 \mathrm{~Hz}$ the best shielding material, was copper.
\end{abstract}

Key words: Efficiency, Frequency, Magnetic Shielding, Magnetic Field, Voltage.

\footnotetext{
${ }^{a}$ Ingeniería Eléctrica, Facultad Tecnológica, Universidad Distrital Francisco José de Caldas, Colombia.

*Autor de correspondencia: jesust07@gmail.com
} 


\section{Introducción}

La incidencia de los campos electromagnéticos ha sido un tema de estudio para diversas aplicaciones en ingeniería, entre las cuales está la protección de equipos electrónicos de estos campos.

Estos dispositivos, al ser sometidos a campos electromagnéticos, se ven afectados por corrientes parásitas cuyo origen se debe a la inducción de ondas electromagnéticas producidas por señales eléctricas de radio y telecomunicaciones; los dispositivos electrónicos más susceptibles ante la presencia de campos electromagnéticos son aquellos utilizados en sistemas de información, debido a que estos campos afectan la fiabilidad de transferencia de datos entre los diferentes sistemas [1].

En particular, para proteger un dispositivo ante la presencia de campos magnéticos se utilizan materiales conductores que permiten reducir los campos producidos por una fuente cercana. Dichos materiales, cuando son sometidos a un campo magnético, crean corrientes en su superficie que generan líneas de campo que se oponen al campo exterior, por lo tanto, reducen la incidencia de los campos magnéticos en su interior.

En diversos estudios sobre blindaje magnético se realizan análisis teóricos teniendo en cuenta el comportamiento de la onda electromagnética al atravesar un material apantallador, pero no se realizan montajes experimentales donde estos materiales sean sometidos a un campo magnético uniforme, para de esta manera comparar los diferentes resultados con los modelos teóricos.

En el trabajo de Oliveras (2010) [2] se realizaron comparaciones de nuevos compuestos derivados del Mumetal (Permalloy ${ }^{\circledR}$, Vacoperm ${ }^{\circledR}$ y Vitrovac ${ }^{\circledR}$ ), los cuales permitieron utilizar unas placas más delgadas en cuanto a los materiales convencionales para evaluar la eficiencia del apantallamiento para estaciones transformadoras, comparando el modelo teórico desarrollado con el propuesto por el investigador Heinrich Von Kaden, donde se tiene en cuenta materiales con alta permeabilidad magnética, pero el análisis era estrictamente teórico, es decir, sin realizar montajes experimentales.

En el estudio de Ren et al. (1995) [3] se indica el cálculo de la eficiencia de apantallamiento, realizando un modelo que utiliza una bobina que encierra una placa metálica con un núcleo magnético, donde se obtuvo como resultado las variaciones de tensión, las cuales se midieron a la salida con y sin blindaje magnético, donde no se observa montaje experimental que mide el modelo.

Por otra parte, en el estudio de Saadi (2007) [4] sobre apantallamiento se realizó un análisis teórico de la eficiencia de blindaje magnético con diversos materiales, donde se tienen en cuenta las variables de reflexión y absorción de estos y su respuesta cuando son sometidos a la influencia de ondas electromagnéticas y verifican su eficiencia por medio de resultados del software Matlab ${ }^{\circledR}$. En este estudio se incluyen las características de los materiales, considerándolos ideales, donde se evidencia la necesidad de comparar con datos obtenidos por una práctica donde se evalue qué tan aproximados son los datos arrojados por el software con los datos reales.

En el estudio de Sánchez (2010), en su tesis de maestría [5], se realiza un análisis teórico que se modela mediante un circuito eléctrico para representar el acople magnético entre los diferentes elementos del montaje, donde los materiales utilizados son hierro y aluminio. Surge la inquietud de cómo es el comportamiento de otro tipo de material, como el cobre y el acero inoxidable, que son muy comunes en el sector industrial, ante la presencia de campos magnéticos de origen externo.

Con base en este tipo de prácticas, se decide realizar un estudio teórico, simulaciones y, finalmente, un montaje experimental diseñado para comparar la eficiencia de los materiales para apantallar ante campos magnéticos, observando de esta manera cómo se comportan las diferentes características de los materiales en respuesta a un campo aplicado y la eficiencia de blindaje magnético.

Con el fin de generar campos magnéticos uniformes para el estudio de la eficiencia de apantallamiento (blindaje magnético) en materiales conductores, se utilizó una bobina que encierra tanto el material apantallador como la bobina sensor (Ver figura 1) [6]. Para medir el campo magnético de forma indirecta se utiliza una bobina sensor que está ubicada en el centro de la bobina.

Finalmente, para verificar la eficiencia de apantallamiento de los materiales ante campos magnéticos, se construye un encerramiento cúbico del material 


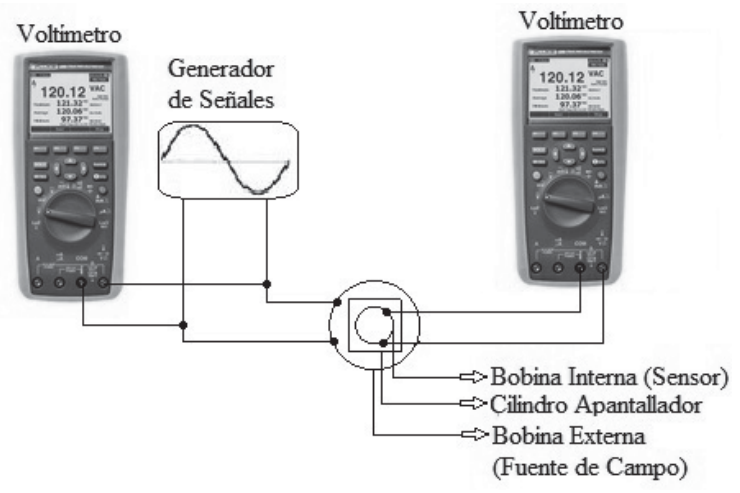

Figura 1. Esquema alimentación bobina externa.

para la bobina sensor, la cual mide la atenuación del campo magnético mediante la disminución de la tensión inducida en ella [5]. La eficiencia del material se ve afectada por la permeabilidad, la conductividad y el espesor del material, además de la frecuencia de la fuente de alimentación de la bobina que genera el campo [7].

A continuación se presenta la metodología desarrollada en el estudio de apantallamiento magnético.

\section{Metodología}

El estudio de apantallamiento se llevó a cabo mediante los siguientes pasos:

- Generación de la señal sinusoidal para la alimentación de la bobina externa.

- Creación de campo magnético uniforme en la bobina externa.

- Comparación de tensión inducida en el sensor con y sin blindaje magnético.

- Medición de las señales de salida con y sin blindaje.

- Cálculo de la eficiencia del blindaje.

\subsection{Montaje}

Para generar un campo magnético uniforme, se utilizó una bobina externa alimentada por un generador; el esquema de conexión de las bobinas se detalla en la figura 1.

El generador se trabajó a su tensión máxima de 10 [Vp], con el objetivo de garantizar la mayor densidad de flujo magnético posible en la bobina externa,con un rango de frecuencias desde 60 hasta 100.000 [Hz]. La impedancia interna del generador es $50[\Omega]$.
De esta forma, la corriente que fluye en la bobina externa tiene la forma:

$$
I_{H}=\frac{V_{G}}{R_{i}+R_{H}+j \omega L_{H}}
$$

Donde:

$I_{H}$ es la corriente bobina externa

$V_{g}$ es la tensión generador de señales

$R_{H}$ es la resistencia bobina externa

$R_{i}$ es la resistencia interna del generador

$L_{H}$ es la inductancia bobina externa

$\omega$ es la frecuencia angular del generador

La densidad de campo magnético en la bobina externa puede ser hallada a partir de la ley de BiotSavart [7], y expresada mediante la ecuación:

$$
B=\mu_{0}\left(\frac{4}{5}\right)^{\frac{3}{2}} \frac{N I_{H}}{R}
$$

Donde:

$B$ es la densidad de campo magnético

$R$ es el radio bobina externa

$N$ es el número de vueltas

$I_{H}$ es la corriente bobina externa

$\mu_{0}$ es la permeabilidad del vacío.

Para calcular la tensión inducida en la bobina sensor se aplica la ley de inducción de Faraday [4], encontrando:

$$
V_{\text {inducida }}=N_{\text {sensor }} \omega \theta_{B}
$$

Donde:

$V_{\text {inducida }}$ es la tensión inducida en el sensor

$N_{\text {sensor }}$ es el número de vueltas bobina sensor

$\omega$ es la frecuencia angular de la señal alterna

$\theta_{B}=B A_{\text {sensor }}$, donde $B$ es la densidad de flujo magnético hallada previamente, con $A_{\text {sensor }}$, como el área transversal del sensor.

\subsection{Materiales apantalladores}

Los materiales utilizados son hierro (con aproximadamente $4 \%$ de Carbono y $2 \%$ de Silicio), aluminio (con bajo porcentaje de cromo y manganeso), cobre y acero inoxidable con contenido inferior de 
Níquel menor al 2,5\%, con un grosor promedio de $1 \mathrm{~mm}$; estos materiales fueron escogidos debido a que son comunes en el sector eléctrico y de telecomunicaciones [8]. A partir de estos, se construyeron cubos con una arista aproximada de $7 \mathrm{~cm}$.

\subsection{Eficiencia del blindaje}

Para el cálculo de la eficiencia del blindaje, se tuvieron en cuenta los resultados obtenidos a partir del montaje, el software de simulación y el desarrollo analítico; en este último, se trabaja el campo magnético como una onda electromagnética que atraviesa un material que presenta una respuesta de absorción y reflexión [5], de esta manera se determina la eficiencia total como función de la absorción, la reflexión y las reflexiones múltiples de acuerdo con [9]:

$$
E F T=A+R+B
$$

con la absorción del material expresada como:

$$
A=131,4 g \sqrt{\mu_{r} \sigma_{r} f}
$$

y el factor debido a la reflexión especular:

$$
R=14,16+10 \log _{10}\left(\frac{r^{2} f \sigma_{r}}{\mu_{r}}\right)
$$

finalmente, la relación para las reflexiones múltiples:

$$
B=20 \log _{10}\left(1-\exp \frac{-2 g}{\delta}\right)
$$

Con: $\mu_{r}$ la permeabilidad relativa

$g$ el espesor material

$\sigma_{r}$ la conductividad relativa

$f$ la frecuencia de fuente.

$r$ la distancia desde del campo magnético al blindaje.

La profundidad de penetración del campo, en pulgadas, es dada por la expresión:

$$
\delta=\frac{2,6}{\sqrt{f \mu_{r} \sigma_{r}}}
$$

La eficiencia del blindaje depende proporcionalmente del efecto piel de material [3]:

$$
S_{d B} \propto \frac{1}{\delta}
$$

Por lo tanto, considerando solamente las características del material [2]:

$$
S_{d B} \propto \mu \sigma
$$

Tabla 1. Características eléctricas de los materiales utilizados en este trabajo.

\begin{tabular}{cccc}
\hline Material & $\sigma_{r}$ & Unidad & $\mu_{r}$ \\
\hline Acero & $0,116 \mathrm{E}+7$ & {$[\mathrm{~S} / \mathrm{m}]$} & 1.075 \\
Aluminio & $3,77 \mathrm{E}+7$ & {$[\mathrm{~S} / \mathrm{m}]$} & 1.000023 \\
Cobre & $5,81 \mathrm{E}+7$ & {$[\mathrm{~S} / \mathrm{m}]$} & 0,999999 \\
Hierro & $1,12 \mathrm{E}+7$ & {$[\mathrm{~S} / \mathrm{m}]$} & 4000 \\
\hline
\end{tabular}

\section{Resultados y discusiones}

En las figuras que se muestran a continuación se realiza la comparación de los datos medidos en el laboratorio, y el modelo. Se tomaron 56 datos por cada material de la siguiente manera: en pasos de $200 \mathrm{~Hz}$ desde 200 hasta $1.000 \mathrm{~Hz}$, luego en pasos de 2.000 hertz hasta $100.000 \mathrm{~Hz}$.

El paso escogido permite observar el comportamiento de la eficiencia de manera que el sistema no experimente cambios bruscos en respuesta a la frecuencia de la señal externa.

Cabe destacar que se realizó el análisis para el hierro a partir de los datos tomados del laboratorio y comparados con los obtenidos en el análisis teórico y en las simulaciones, donde no se observa que la permeabilidad alcanza su máximo valor; razón por la cual los resultados para este material discrepan para cada uno de los análisis $[10,11]$.

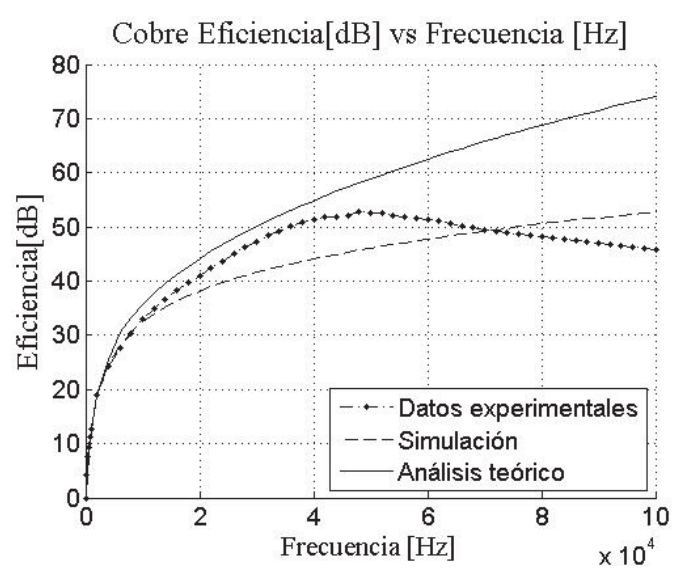

Figura 2. Curva de Eficiencia $[\mathrm{dB}]$ vs. Frecuencia $[\mathrm{Hz}]$ para el Cobre de 60 a $100.000 \mathrm{~Hz}$.

En la figura 2 se observa cómo la eficiencia del cobre tiene un comportamiento creciente hasta cerca de los $50.000 \mathrm{~Hz}$, punto en el cual empieza a descender levemente hasta llegar a los $100.000 \mathrm{~Hz}$; este comportamiento se debe al efecto piel producido por las altas frecuencias a las cuales se somete el material $[12,13]$; esto hace que la resistencia aumente $y$, por 


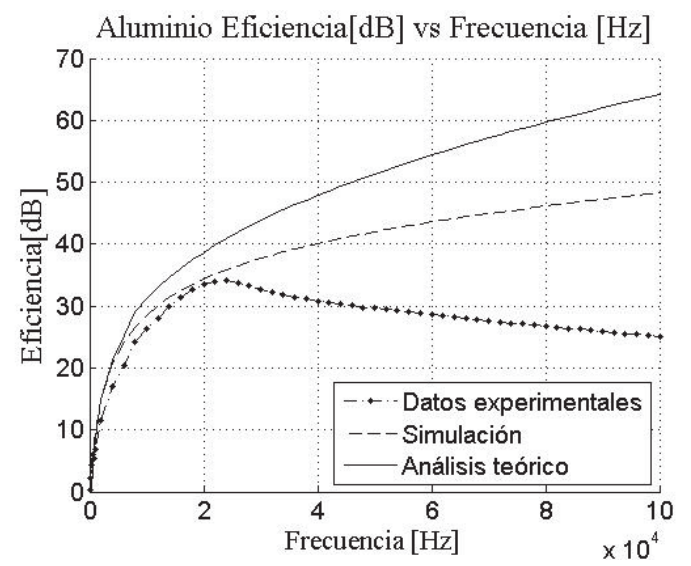

Figura 3. Curva de Eficiencia $[\mathrm{dB}]$ vs Frecuencia $[\mathrm{Hz}]$ para el Aluminio de 60 a $100.000 \mathrm{~Hz}$.

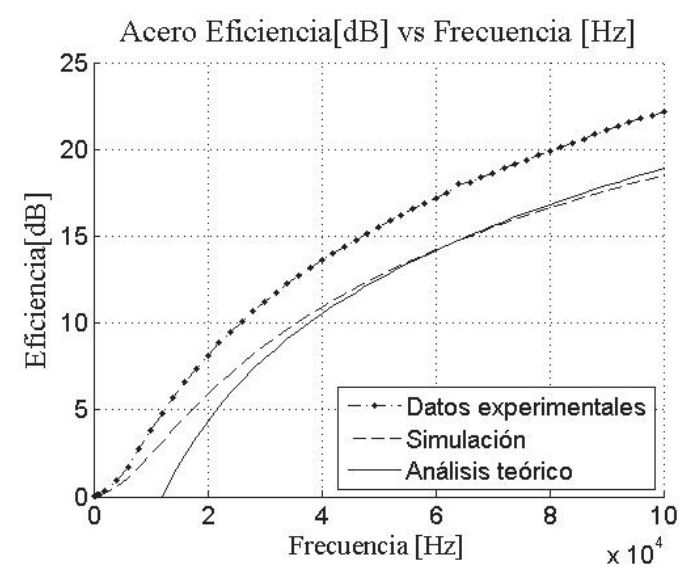

Figura 4. Curva de Eficiencia [dB] vs. Frecuencia $[\mathrm{Hz}]$ para el Acero de 60 a $100.000 \mathrm{~Hz}$.

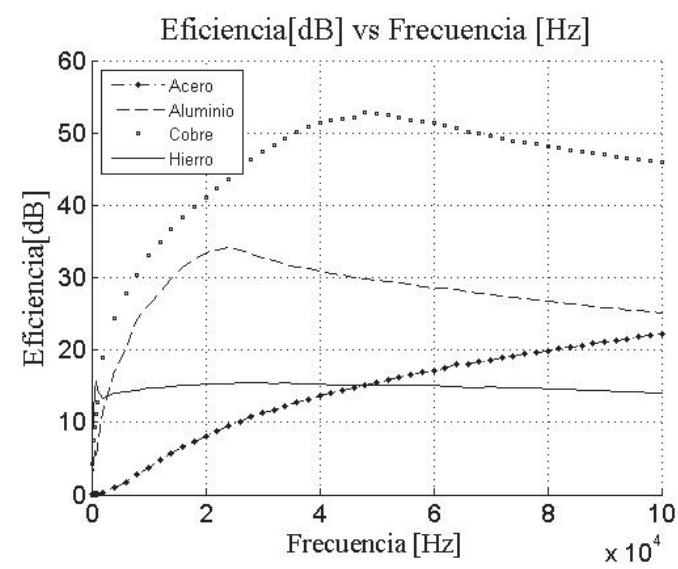

Figura 5. Curva de Eficiencia $[\mathrm{dB}]$ vs. Frecuencia $[\mathrm{Hz}]$ para acero, hierro, cobre, aluminio 60 a $100.000 \mathrm{~Hz}-$ Datos experimentales.

lo tanto, la corriente tienda a circular por la periferia del material, provocando que la eficiencia de apantallamiento se vea disminuida. Lo mismo sucede

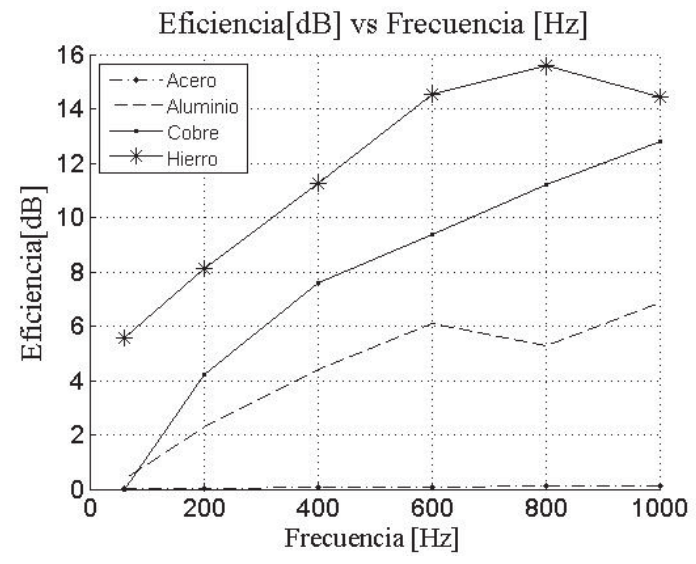

Figura 6. Curva de Eficiencia $[\mathrm{dB}]$ vs. Frecuencia $[\mathrm{Hz}]$ para el acero de $60 \mathrm{a} 1000 \mathrm{~Hz}$.

para el aluminio en la figura 3, pero este descenso se presenta cerca a los $20.000 \mathrm{~Hz}$ [14].

Por otra parte, el comportamiento de la eficiencia del acero es similar tanto a la simulación como al análisis teórico; este comportamiento ascendente que se observa hasta los $100.000 \mathrm{~Hz}$ se debe a que este material presenta la conductividad más baja; por lo tanto, el efecto piel es mayor y la corriente no presenta el mismo comportamiento como en el caso del cobre y el aluminio [15].

En las figuras 5 y 6 se observa la comparación de la eficiencia del blindaje magnético para los materiales utilizados en el laboratorio, en donde se comprueba que la eficiencia depende proporcionalmente de la conductividad y permeabilidad del material, puesto que los materiales más eficientes son el hierro y el cobre $[16,17]$.

Además, se observa la comparación de la eficiencia del blindaje magnético para los materiales utilizados en el laboratorio, en donde se comprueba que la eficiencia depende proporcionalmente de la conductividad y permeabilidad del material, puesto que los materiales más eficientes son el hierro y el cobre [18].

En bajas frecuencias, el hierro es el material que mejor apantalla el campo magnético, debido a su alta permeabilidad.

Para el caso del acero, el cobre y el aluminio, que tienen una permeabilidad magnética cercana a uno (1) se comprueba lo estipulado en la tabla 1, en donde la eficiencia crece proporcionalmente debido a la conductividad del material [19]. Aunque el hierro 
presenta una baja conductividad respecto al cobre y al aluminio, manifiesta una eficiencia similar a la de estos dos materiales, debido a su alta permeabilidad.

En la figura 7 se observa un plano tangencial a las bobinas, el cual describe el comportamiento del campo magnético obtenido mediante el software de simulación Comsol Multiphysics ${ }^{\circledR}$, en donde es posible detallar el efecto del cubo apantallador en la disminución de dicho campo [13].

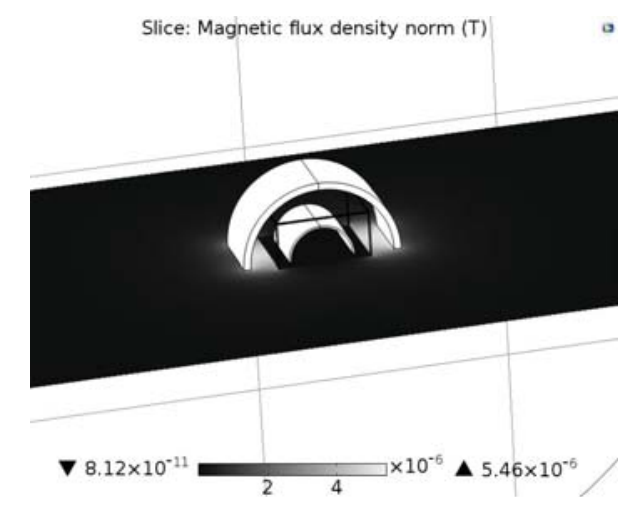

Figura 7. Gráfica de campo magnético con apantallamiento- Obtenida del software de simulación Comsol Multiphysicss ${ }^{\circledR}$.

\section{Conclusiones}

Al comparar los diferentes materiales en las pruebas realizadas, se concluye que el cobre, al ser un material diamagnético, orienta sus momentos magnéticos en sentido contrario del campo magnético externo, obteniendo un comportamiento óptimo en cuanto al blindaje magnético en el rango de frecuencias de 1.000 a $100.000 \mathrm{~Hz}$.

El hierro tiene un comportamiento diferente a los demás materiales objeto del estudio, debido a su propiedad de alta permeabilidad magnética e hist resis. Este comportamiento ocurre debido a que la permeabilidad varía en función de la frecuencia del campo magnético al cual está sometido el material.

Los resultados obtenidos en el laboratorio son comparables con el desarrollo analítico y la simulación, con algunas diferencias, debido a que estos análisis manejan una pureza del material diferente a la utilizada en el laboratorio. Por otra parte, el análisis teórico no tiene en cuenta la geometría del blindaje y las conexiones eléctricas [16].

Los materiales utilizados en el laboratorio, a diferencia del acero, presentan saturación debido a su respuesta dipolar magnética frente a la señal de alta frecuencia, la cual provoca que la eficiencia descienda [20].

Se comprueba la importancia de realizar diferentes estudios sobre blindajes magnéticos, puesto que en el análisis teórico y la simulación la eficiencia crece indefinidamente en función de la frecuencia sin saturarse, puesto que las variables eléctricas son consideradas constantes, obteniendo discrepancias con los datos experimentales.

A futuro, es necesario realizar el estudio de blindaje magnético con otros materiales, como níquel, bronce, mumetal y permaloy.

\section{Referencias}

[1] C. F. Barbosa, and F.E. Nallin, "Protection of Telecommunication Equipment from Nearby Lightning Magnetic Field", International Symposium on Lightning Protection, vol. 11, no. 7, pp. 58-61, Brazil, October 3-7, 2011.

[2] J. M. Oliveras, "Blindaje magnético de estaciones transformadoras", Escuela Politécnica de Cartagena. Octubre, 2010, Disponible en: http://www.tecnicaindustrial.es/TIAdmin/ Números/74/408/a408.pdf

[3] S. Ren, H. Ding, M. Li, and S. She, "Magnetic Shielding Effectiveness for Comparators", IEEE Transactions on Instrumentation and Measurement, vol. 44, no. 2, pp. 422-424, Abril 1995.

[4] H. Saadi, and R. Oussaid, "Materials Effect On Shielding Effectiveness", IEEE International Conference on Signal Processing and Communications (ICSPC 2007), pp. 992-1002, Dubai, United Arab Emirates, November 2007.

[5] A. Sánchez, "Estudio de materiales metálicos usados como apantallamiento de campos magnéticos", Tesis de Maestría, Fac de Ingeniería, Dep. Ingeniería Eléctrica y Electrónica, Universidad Nacional de Colombia, sede Bogotá, Colombia, 2010.

[6] P. Clayton, "Shielding", Introduction to electromagnetic compatibility, 2nd Edition. J. Wiley and Sons, New York, pp 713-742, 2006.

[7] H. Ott, "Shielding", Electromagnetic Compatibility Engineering, 2nd Edition, J. Wiley and Sons, New York, pp. 238-265, 2009. 
[8] R. Oussaid, "The electromagnetic shielding depending on material?s parameters", Instrumentation Laboratory, F E I Houar Boumediène, University OF Sciences and Technology, Algiers, Algeria, 2010.

[9] R. W. Evans,, "Design Guidelines for Shielding Effectiveness, Current Carrying Capability, and the Enhancementof Conductivity of Composite Materials", NASA, Alabama, Marshall Space Fight Center, Agosto, 1997, pp. 19-36.

[10] A. Charles, and S. Matthew, "Respuestas en frecuencia", Fundamentos de circuitos eléctricos, 2nd Edition, Editorial McGraw-Hill, México, pp. 637-642, 2006.

[11] X. Liu, C. Gao, X. N. Chen, H. Wu, L. H. Shi, and B. H. Zhou, "Portable Measuring Equipment for Extremely Low Frequency Magnetic Field Shielding Effectiveness", Lab of Electromagnetics, Nanjing Engineering Institute, no. 1 Haifuxiang, Nanjing, China, pp. 978-981, 2008.

[12] A. Keshtkar, A. Maghoul, and A. Kalantarnia, "Magnetic Shield Effectiveness in Low Frequency", International Journal of Computer and Electrical Engineering, vol. 3, No. 4, pp. 507-513, August 2011.

[13] J. Cuntala, "Simulation Of Electromagnetic ShieldingIn Comsol Multiphysics Environment", Department of mechatronics and electronics, Faculty of Electrical Engineering, University of ?ilin, 2006.

[14] J. Alarcón, and M. Albaladejo, "Ciclo de Histéresis", Licenciatura en Física, Universidad de Murcia, Junio 2004. Disponible en: http://www.fisimur.org/wpcontent/uploads/histeresis-articulo.pdf.
[15] X. Di, "Magnetic shielding using electrical steel panels at extremely low frequencies", Tesis Doctoral,Wolfson Centre for Magnetics, School of Engineering, Cardiff University, United Kingdom, 2008.

[16] M. Kühn, W. John, and R.Weigel, "Validation of a measurement method for magnetic shielding effectiveness of a wire mesh enclosure with comparison to an analytical model", Adv. Radio Science, Vol 11, pp. 189?195, 2013, Disponible en: http://www.adv-radiosci.net/11/189/2013/ars-11-189-2013.pdf.

[17] P. L. Arnera, M. B. Barbieri, D. A. Esteban, N. A. Casco, and J. E. Conti, "Efectividad del blindaje de campo magnético de baja frecuencia, con placas metálicas", Congreso Internacional de Distribución Eléctrica, Instituto de Investigaciones Tecnológicas para Redes y Equipos Eléctricos (IITREE). Facultad de IngenieríaUniversidad Nacional de La Plata, Argentina, 2006.

[18] Z. Yichao, G. Cheng, S. Lihua, and Z. Bihua, "Analysis and Test of EM Shielding for LowFrequency Magnetic Field", Engineering Institute of Engineering Corps, PLA University of Science and Technology, Nanjing, China, pp. 345-349 2007.

[19] S. J. Ferrara, and H. D. Reisin, "Estudio del blindaje magnético y de las corrientes de Foucault", Facultad de Ciencias Exactas y Naturales, Universidad de Buenos Aires, Buenos Aires, Argentina, pp. 1-13, 2004.

[20] A. Frikha, M. Bensetti, F. Duval, F. Lafon, and L. Pichon, "Modeling of the Shielding Effectiveness of Enclosures in Near", Université Pierre et Marie Curie, pp. 612-615, 2003. 